$$
\begin{aligned}
a_{2}^{3} & \left.+\left(-\frac{51}{4 \cdot 5} a_{1}+3 a_{3}\right) a_{2}^{2}+\left\{\frac{5}{4} a_{1}^{4}+\frac{1}{4} a_{2} a_{1}^{3}-\frac{287}{6} a_{1}^{2}+\left(-\alpha_{3}+3 \alpha_{4}\right) a_{1}\right)\right\} a_{-2} \\
& -\frac{1}{2 \cdot 3 \cdot 7 \cdot 8} a_{1}^{7}+\frac{31}{3 \cdot 4 \cdot 5} a_{1}^{5}+\left(-\frac{1}{3} \alpha_{2}+\frac{1}{4} a_{3}\right) a_{1}^{4}-\frac{839}{5 \cdot 8} a_{1}^{3}-\left(-\frac{91}{2 \cdot 5} \alpha_{3}+4 a_{4}\right) a_{1}^{2} \\
& +\left(147+\frac{3}{2} a_{3}^{2}-46 \cdot 7 \frac{y_{l}}{l}\right) a_{1}-6\left(1-a_{0}\right)=0,
\end{aligned}
$$

The roots of these two simultaneous equations constitute the solution of the problem.

As examples, we will take for the rings the ellipse of semi-axes 2 and 1 and a circle of radius 1 as in my former paper cited above.

The result of calculation is, supposing the elongation to be five per cent in each case,

$$
\left.\begin{array}{l}
a_{1}=-.72 \\
a_{2}=.15 \\
a_{1}=-1.89 \\
a_{2}=.50
\end{array}\right\} \text { for the ellipse, }
$$

These figures were spotted out graphically by using $20 \mathrm{~cm}$ by $26 \mathrm{~cm}$ section paper, and must be considered as rough indications of their whereabouts.
Using these figures, we get

$$
\begin{aligned}
& \begin{array}{l}
B=.42 . \\
C=.12 .
\end{array} \\
& \begin{array}{l}
\text { For non-studded chain } \\
B=.41 .
\end{array} \\
& \begin{array}{l}
B=.29 . \\
C=1.00 .
\end{array} \\
& \left.\begin{array}{l}
\text { For non-studded chain } \\
B=.637 .
\end{array}\right\} \text { for the ellipse, }
\end{aligned}
$$

The general conclusions and recommendations in my former paper remain unchanged.

\section{Reference}

Yokota, S.: Strength of a Studded Chain Cable, Jonrnal of the Japan Society for Applied Mechanics, Vol. 3, No. 19, October, 1950, pp. 168-171.

\title{
Strength of a Studded Chain Cable (ter) ${ }^{*}$
}

\section{By Late Seinen YокотA ${ }^{* *}$}

To verify the calculated results by means of model experiments, the following three forms of chain rings were adopted:

A circle of $20 \mathrm{~cm}$. diameter,

An ellipse of axes $20 \mathrm{~cm}$. and $30 \mathrm{~cm}$.,

An ellipse of axes $20 \mathrm{~cm}$. and $40 \mathrm{~cm}$.

The calculation was gone over anew, and the models were prepared as will be described shortly.

The constants $a_{s}$ were calculated by using the values of $\theta$ at equal intervals in the Gudermannian amplitude, namely, at $22.5^{\circ}, 45^{\circ}, 67.5^{\circ}$ and $90^{\circ}$. The constant $\alpha_{1}$ being known beforehand, they give $\alpha_{2}, \alpha_{3}, \alpha_{4}$ and $\alpha_{5}$ for each ellipse. The calculated $\alpha_{s}$ are

\begin{tabular}{lccccc} 
& $\alpha_{1}$ & $\alpha_{2}$ & $\alpha_{3}$ & $\alpha_{4}$ & \multicolumn{1}{c}{$\alpha_{5}$} \\
Circle $\left(20 \mathrm{~cm} . \mathrm{dia}_{2}\right)$ & -1.571 & 0 & 0 & 0 & 0 \\
Ellipse $(20 \mathrm{~cm} . \times 30 \mathrm{~cm})$. & -0.881 & -0.116 & 0.131 & -0.09335 & 0.229 \\
Ellipse $(20 \mathrm{~cm} . \times 40 \mathrm{~cm})$. & -0.6055 & 0.107 & -1.162 & 2.063 & -1.975
\end{tabular}

As far as I remember, the $\alpha_{s}$ for the last ellipse as are given in my former papers were calculated from values at equal intervals along the perimeter, and differ somewhat from the values given above. But, our calculation being based on the aggregate of $\alpha_{s}$, there ought to be no appreciable difference in the result whether we use one set or the other.

The models for the experiment are a circular ring and two elliptic rings with the principal dimensions above specified, of uniform crosssection $4 \mathrm{~mm} . \times 4 \mathrm{~mm}$, carefully cut and finished out of polished mild steel plates $4 \mathrm{~mm}$. thick.

The plates were selected, as far as possible, to ensure uniformity of material and elastic property at all spots and directions. The effect of welding of joints and overstraining of material during the preparation of models were thus completely eliminated.

- Received Sept. 4, 1951

** University of Tokyo 
Each ring was suspended from the ceiling with a cord, its major axis being kept vertical. To its lower end a weight of $4.5 \mathrm{~kg}$. was applied. This caused an appreciable lateral contraction of the ring along the minor axis amounting to a few $\mathrm{mm}$.

The ring was then pulled out laterally along the minor axis until it resumed its initial length before the loading, while the corresponding force was measured by a spring balance.

The results of the calculations and the experiments are summarized below:

\begin{tabular}{|c|c|c|c|c|c|c|c|}
\hline \multirow{3}{*}{$\begin{array}{l}\text { Circle } \\
(20 \mathrm{~cm} . \text { dia. }) \\
\text { Ellipse }\end{array}$} & & \multicolumn{3}{|c|}{ Calculated } & \multicolumn{3}{|c|}{ Experimeated } \\
\hline & $a_{1}$ & $a_{2}$ & $B$ & 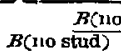 & $\frac{\text { o stud }}{\boldsymbol{B}}$ & & \\
\hline & -1.90 & 0.5 & 0.30 & 0.64 & 2.1 & 1 & 1 \\
\hline $\begin{array}{l}\text { Enpse } \\
(20 \mathrm{~cm} . \times 30 \mathrm{~cm} .) \\
\text { Ellipse }\end{array}$ & -1.16 & 0.2 & 0.28 & 0.51 & 1.8 & 0.64 & 0.62 \\
\hline$(20 \mathrm{~cm} \cdot \times 40 \mathrm{~cm})$. & -0.8 & 0.3 & 0.28 & 0.41 & 1.5 & 0.38 & 0.40 \\
\hline
\end{tabular}

Considering the approximate nature of our calculation and allowing for irregularities of elastic property which may exist in the model despite every effort done to avoid them, the agreement between the calculated and experimental results is quite satisfactory.

By means of the foregoing research,' we may find the scantling of the ring of uniform strength for a studded chain. For practical purposes, its wear and, tear being duly taken into consideration, suitable scantling may be assigned. This provides a chain of greater length for equal weight or a lighter chain for equal length when compared with that generally and universally used at the present time.

The above model experiment was made with an allowance from the Minister for Education, out of our government appropriation for the encouragement of scientific researches for the fiscal year 1951, for which the author offers his. grateful thanks.

It is desirable to push the experiments further with actual chains instead of simple models and to finish up with their breaking tests, in order that the practical application of the present research may bear direct and beneficial fruits for the industry in general. (End)

\title{
On Vibration of a Cylindrical Shell, which is Filled with Water.*
}

\author{
By Fumiki Kıтo**
}

\begin{abstract}
The Author has made hydrodynamical study on vibratory motion of a cylindrical shell, in which water is filled, and has obtained the amount of virtual mass of water for the case where both ends of the cylinder are fitted with rigid end-plates.
\end{abstract}

\section{Introduction}

When a cylindrical shell, inside which water is filled, makes a vibratory motion, the water contained inside the shell also makes vibratory motion. The effect of this vibratory motion of water is to increase the apparent mass of the shell and lower its natural frequency of vibration as compared to values when there is no water. This effect is called the virtual mass of water. In the present paper, the Author gives some results of theoretical estimations of this- virtual mass for the case of a vibration of a shell in form of a circular cylinder, both ends of which are left free, or are closed with rigid end-plates.

According to the calculation, the effect of rigid end plates appear as a kind of "end effect", which has significant influence only if the ratio of the length of cylindrical shell to its radius is less than 5 .

2. Solution for the case of a series of cylinders.

Let us consider a cylindrical shell of radius $a$

* Read befóre Meetings on Vibration and Wave-Motion.

Mar. 15, 1953. Received Sept. 28, 1953

** Department of Engineeting, Keio University. 